\title{
Session B
}

\section{Radiative transfer}


N. E. Piskunov, W. W. Weiss, D. F. Gray, eds.

\title{
3D NLTE Radiative Transfer - Current Status and Future Prospects
}

\author{
Mats Carlsson \\ Institute of Theoretical Astrophysics, P.O. Box 1029 Blindern, N-0315 \\ Oslo, Norway
}

\begin{abstract}
The current status and future prospects for methods of 3D NLTE radiative transfer are reviewed. Scaling properties and parallelization issues are discussed. Codes of today are capable of solving the diagnostic problem (given model atmosphere) with atomic models of 20-100 levels making possible detailed 3D NLTE determinations of abundances employing 3D LTE model atmospheres. Calculating consistent 3D NLTE radiation hydrodynamic models is orders of magnitude more demanding computationally but methods exist that give a reasonably correct description of the radiation interactions in a statistical sense.
\end{abstract}

\section{Introduction}

To infer the physical conditions of stars and other astrophysical objects, we are almost exclusively restricted to an analysis of the photons that escape from the medium. Before the photons reach us they have interacted with the matter so that the information about local conditions in the atmosphere has been convolved both in space and in time. The diagnostic deciphering of the information content in the radiation is made complicated by the fact that most plasmas both emit and absorb photons at the same time. In addition, photons can travel large distances thus coupling distant parts of the plasma making the diagnostic problem inherently three-dimensional. The opacity and emissivity of the medium depends on the distribution over energy levels of the emitting atoms and ions which in turn depends on the radiation field. The standard definition of "stellar atmosphere" is the region from which photons can leave and this very fact drives the distribution over energy levels away from local thermodynamic equilibrium (LTE). In general we thus have to solve a 3D non-LTE (NLTE) radiative transfer problem to diagnose stellar atmospheres.

Radiation also plays an important role in determining the structure of stars through the role of radiation in the energy balance and for some objects also in the momentum balance. Velocity fields in the stellar atmosphere Doppler shift the narrow absorption profiles of spectral lines thus affecting the coupling between the radiation and the local thermodynamic properties of the plasma. Radiative losses damp the temperature increase in the compression phase of a hydrodynamic disturbance. There is thus a strong coupling between the hydrodynamics and the radiation in a dynamic atmosphere and we have to treat this coupling self-consistently in 3D to properly model dynamic stellar atmospheres. 
The layout of this contribution is as follows: in Section 2 we formulate the NLTE problem, in Section 3 we discuss methods of solution focusing on the differences between one-dimension and three dimensions, in Section 4 we discuss the problems of calculating 3D NLTE stellar atmospheres, in Section 5 we discuss the diagnostic problem and we end with conclusions in Section 6 .

\section{Formulation of Problem}

We assume a general familiarity with basic radiative transfer theory and will just give an incomplete summary in order to define notation and highlight important aspects. For an excellent and more complete discussion we refer to Mihalas (1978).

For simplicity we assume a static situation. We then have to solve the equations of statistical equilibrium describing the time-independence of the population density of each energy level, $i$ :

$$
\sum_{j \neq i}^{N} n_{j}(\vec{r}) P_{j i}(\vec{r})-n_{i}(\vec{r}) \sum_{j \neq i}^{N} P_{i j}(\vec{r})=0,
$$

where $N$ is the number of energy levels, $\vec{r}$ is the position in the medium, $n_{j}$ is the number density of atoms in energy level $j$ and $P_{i j}$ is the rate from level $i$ to level $j$. The dependence of the energy level populations on the radiation field lies in the rates $P_{i j}$, which for the case of bound-bound transitions can be written as:

$$
P_{i j}(\vec{r})= \begin{cases}A_{i j}+B_{i j} \bar{J}_{i j}(\vec{r})+C_{i j}(\vec{r}), & \text { if } i>j \\ B_{i j} \bar{J}_{i j}(\vec{r})+C_{i j}(\vec{r}), & \text { if } i<j\end{cases}
$$

where $A_{i j}$ is the Einstein coefficient for spontaneous emission and $B_{i j}$ is the Einstein coefficients for absorption $(i<j)$ and stimulated emission $(i>j), C_{i j}$ is the collisional rate and

$$
\bar{J}_{i j}(\vec{r})=\int_{\Omega} \int_{0}^{\infty} \Phi(\nu, \vec{l}, \vec{r}) I(\nu, \vec{l}, \vec{r}) d \nu d \Omega,
$$

where $d \Omega$ is a solid angle element around a ray in direction $\vec{l}, \Phi$ is the profile function (here assumed to be identical for absorption and stimulated emission. This is not correct in cases where partial distribution is important), $\nu$ is frequency and $I$ is the specific, monochromatic intensity in the direction $\vec{l}$.

$\bar{J}$ is the quantity that in the statistical equilibrium equations, through its dependence on the intensity in all directions, is responsible for the global coupling. For an accurate determination of the population densities in a given location it is thus this angle-frequency integrated quantity that has to be accurately determined, not necessarily the specific intensity in all directions. We will return to this point in Section 3.

The specific intensity in a given direction depends on conditions along this ray throughout the atmosphere. This is described by the equation of radiative transfer: 


$$
\frac{d I(\nu, \vec{l}, \vec{r})}{d \tau(\nu, \vec{l}, \vec{r})}=S(\nu, \vec{l}, \vec{r})-I(\nu, \vec{l}, \vec{r})
$$

where $d \tau(\nu, \vec{l}, \vec{r})$ is the monochromatic optical path length along the ray in direction $\vec{l}$ and $S(\nu, \vec{l}, \vec{r})$ is the local source function (defined as emissivity over opacity). Note that this equation is one-dimensional along the ray. The only difference between one-dimensional radiative transfer and 3D radiative transfer lies in the mapping of the 3D local quantities (source function and opacity) to the one-dimensional ray. We will return to this point in Section 3.

The statistical equilibrium equations (equation (1)) are simple but through the $\bar{J}$ terms in the rates they are non-local. Furthermore, the source functions and the optical depths depend on the population densities. We thus have to solve a large set of coupled integro-differential equations that are non-local and non-linear.

\section{Methods of Solution}

Early work on multi-dimensional transfer include studies of radiative transfer in various geometries by the Monte Carlo technique (Avery 1969, Avery \& House 1969, Avery, House, \& Skumanich 1969, House \& Avery 1969), radiative transfer in hexagonal cells using integral methods (Avrett \& Loeser 1971), line transfer in 2D (Cannon 1970), 2D studies of the lower solar chromosphere (Cannon 1971a,b), 2D studies of the transfer in strong lines including frequency and angle redistribution (Cannon 1976), integral methods in multi-dimensional media (Jones 1970, 1971, 1973a,b; Jones \& Skumanich 1973) and core saturation techniques applied to cylindrical magnetic flux tubes in the solar atmosphere (Stenholm 1977, Stenholm \& Stenflo 1977, 1978). Mihalas, Auer, \& Mihalas (1978) developed efficient techniques based on the differential equation form of the transfer equation and discussed several 2D examples of periodic media and free standing slabs including effects of velocity fields.

The first 3D NLTE spectral line formation study with a realistic 3D model atmosphere was presented by Nordlund (1985). He developed a method to solve the two-level atom with overlapping continuum based on treating the radiative transfer along a number of rays through the model as separate sub-problems combining the individual estimates of the correction needed such that the converged solution is the correct solution to the problem. The method was applied to the study of an iron line in a solar atmosphere represented by a snapshot from a convection simulation. He found that the granular velocity field has a substantial influence on the behaviour of the line source function in the upper photosphere. The method was used by Kiselman \& Nordlund (1995) in a study of the 3D NLTE line formation of the OI $777 \mathrm{~nm}$ triplet lines in the solar atmosphere. A similar method of angle-decoupling of the rays was used by Skartlien (1998) who developed an efficient method to treat coherent scattering problems in $3 \mathrm{D}$, suitable for inclusion in radiation hydrodynamic codes.

As was pointed out in the previous section there is not much difference between 1D and 3D methods of solution since the main work consists of solving 
the equation of radiative transfer along the path of the photons. Advances in $1 \mathrm{D}$ methods can thus often be carried over to 3D.

Methods in radiative transfer in 1D applied to stellar atmospheres are now dominated by accelerated (or approximated) $\Lambda$-iteration (ALI) techniques. These techniques are based on operator splitting, first used in this context by Cannon (1973). For reviews on $\Lambda$-iteration techniques, see Hubeny (1992) and several contributions in Hubeny, Mihalas, \& Werner (2003). The basic ingredients in these ALI techniques is that the error of the current iteration is evaluated accurately in the so called formal solution step (where all population densities, source functions, opacities etc are known). The corrections are then evaluated using an approximation of the full $\Lambda$ operator. Different choices of approximations give different methods. Some possible choices are the global operator of Scharmer (1981), giving very rapid convergence but limited to 1D and small model atoms; the diagonal of the full $\Lambda$ operator (Olson, Auer, \& Buchler 1986, Rybicki \& Hummer 1991) excellent for multi-dimensional work; a banded approximate matrix (Olson \& Kunasz 1987, Rybicki \& Hummer 1991) often optimal for large 1D problems.

In $1 \mathrm{D}$ it is possible to take advantage of the structure of the approximate operator; a sparse matrix with very few underdiagonal elements in the Scharmer operator or the tri-diagonal or penta-diagonal structure in the banded operators. In $2 \mathrm{D}$ or $3 \mathrm{D}$ the sparseness is spread over the full matrix and the inversion of a non-local approximate $\Lambda$ operator becomes prohibitive even with methods of inverting sparse matrices. Using the diagonal of the exact $\Lambda$ operator as approximation is equivalent to Jacobi iterations. Trujillo Bueno \& Fabiani Bendicho (1995) combined the efficiency of local methods with the fast convergence of global methods in a Gauss-Seidel iteration method giving optimal performance.

In $3 \mathrm{D}$ there are two dominant methods to perform the formal solution; the long characteristics method (of which the Feautrier (1964) scheme is one example) and the short characteristics method. In the long characteristics method rays traverse the whole computational box. One can shoot rays through all gridpoints (see Figure 1, left panel) interpolating source functions and opacities onto the rays but obtaining the solution at each grid-point with no interpolations of the solution back to the grid. This is obviously very costly and the solution along the rays that has to be calculated as part of the process is not used. The alternative approach is to let the rays go through only one plane of grid-points (e.g. around optical depth unity) (Figure 1, right panel). The source functions and opacities are then interpolated onto the rays in each plane, the $1 \mathrm{D}$ radiative transfer problem is solved along each ray and the resulting contribution to the rate (and radiative heating/cooling in the structure problem) is interpolated back to the atmospheric grid. All 1D solutions are independent and would parallelize very well but the problem is that all CPUs then need the full atmospheric grid as well as the approximate lambda matrices at all grid points; quickly an overwhelming amount of storage. In the method of short characteristics (Kunasz \& Auer 1988) the transfer equation is integrated over one grid cell at a time keeping the problem much more local (Figure 2). This is an advantage in parallelization since the computational grid can be distributed over processors. The disadvantage is that a processor integrating a part of the ray needs the boundary value before it can progress. The penalty of this dependency can be 


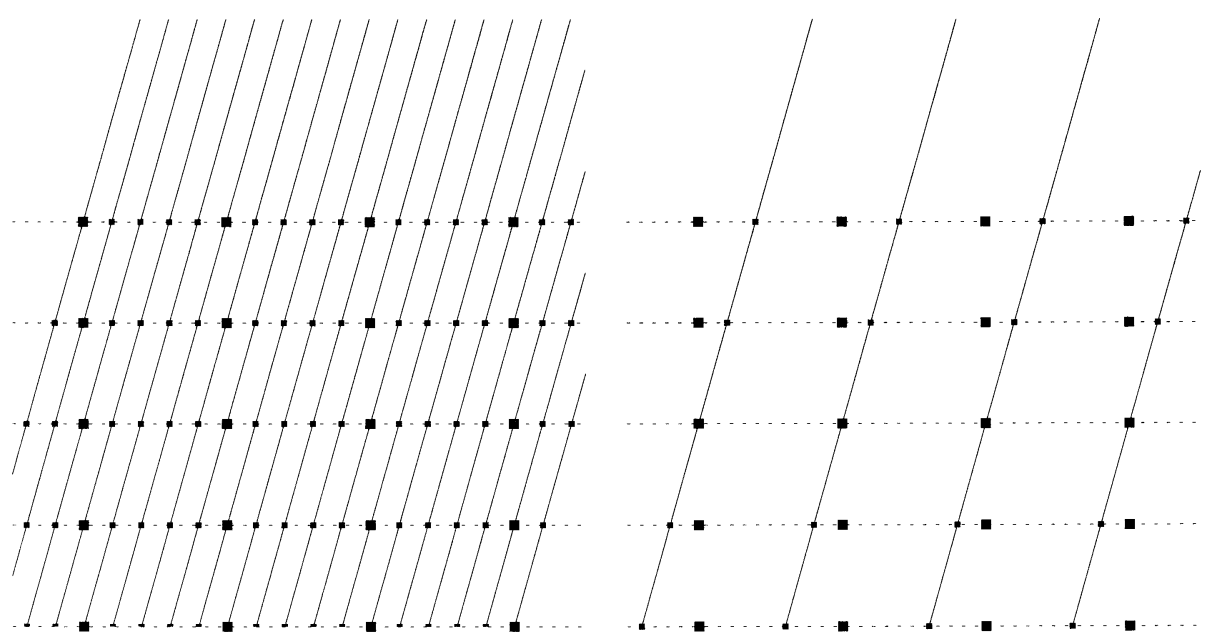

Figure 1. Long characteristics in 2D. The grid-points are shown as large black squares, grid-planes with dotted lines, rays with solid lines and intersections of rays and grid-planes with small black squares. Left panel shows a situation where rays go through all computational gridpoints, the right panel shows rays going through the grid-points in one plane only

minimized by arranging the computations such that a processor starts working as soon as the boundary data needed has been produced but with all processors working on different rays at any given time. This will work well if the number of rays is very large (see Nakamoto 1999). Another approach is to iteratively update the boundary data. This approach often converges fast because in most regions the radiation is either optically thick (and thus locally determined) or free streaming. For a discussion of implementations of the short characteristics method on a regular grid on a massively parallel computer, see Väth (1994).

The formulations above are on Cartesian grids. Van Noort, Hubeny, \& Lanz (2002) have developed a formulation of the short characteristic method in a universal way so it can be used in Cartesian, cylindrical or spherical geometries. They also address how to deal with large opacity gradients along the short characteristic in the presence of velocity gradients (Doppler shifts of narrow line profiles) and domain decomposition parallelization issues.

A potential problem with the ALI methods is that the number of iterations go up when the grid resolution increases. The reason for this is that the lowfrequency spatial components of the error take many iterations to propagate over the fine grid. In $3 \mathrm{D}$ one often has large gradients locally in the computational domain. Without local mesh refinement, the resolution of the gradient in these regions leads to an unnecessarily fine grid in other areas leading to slow error propagation of the low-frequency component. A way to escape this problem is to employ multigrid methods (Brandt 1977, Hackbush 1985, Steiner 1991, Väth 1994) where the problem is solved on a series of grids where the solution on the finest grid takes care of the high-frequency component of the error and the 

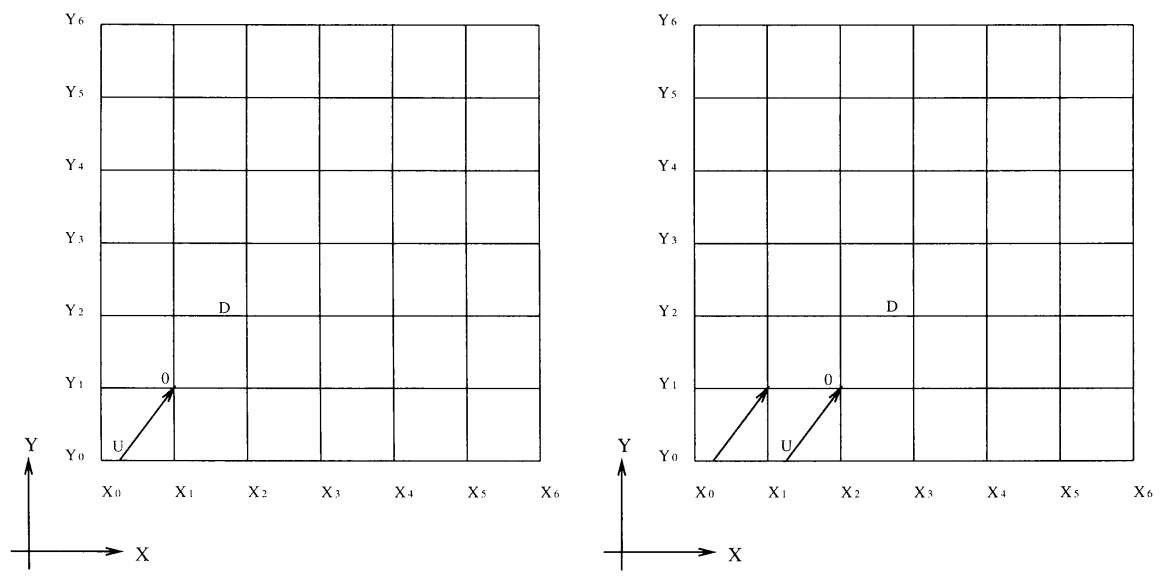

Figure 2. Short characteristics in 2D. The intensity in point 0 is calculated from the analytic integral of a polynomial fit of the source function through points $\mathrm{U}, 0$ and $\mathrm{D}$ and the intensity at point $\mathrm{U}$

solution on the coarsest grid of the low-frequency component. Fabiani Bendicho, Trujillo Bueno, \& Auer (1997) have developed a nested multigrid radiative transfer code where the number of iterations is insensitive to the grid resolution. Since the computational cost per iteration is higher than in standard ALI schemes these methods are primarily of interest when the grid size is small. The saving is larger for $3 \mathrm{D}$ applications than for 2D or 1D.

Any finite difference method has to resolve all important quantities in the discretization in order to produce reasonably accurate solutions. Resolution in space means enough grid-points in the proper locations to get a decent resolution in optical depth along all rays. This is an issue of concern already in 1D and a formidable challenge in 3D since we may have very much sharper population density (and therefore opacity) gradients than temperature gradients. Apart from accuracy problems in cases of large unresolved gradients we may get convergence problems and interpolation overshoot problems for higher order methods. This is illustrated in Figure 3 for the case of the short characteristic method. Several methods exist that address this interpolation problem, see Auer (2002a).

Finite difference methods do not only discretize in space but also in angle. As pointed out in Section 2 the important quantity is $\bar{J}$. At each point, the contributions to $\bar{J}$ have to be included in the angle discretization. In studies of the reionization of the universe one is faced with a situation with lots of high density clumps embedded in an optically thin environment. Every clump has to "see" every other clump in order for the rates to be properly calculated and this means an enormous number of rays. Nakamoto (1999) employed $128 \times 128$ rays in a computational box of $128^{3}$ points and 6 frequencies to address this problem.

In the case of a stellar atmosphere the angle resolution poses much less of a problem because of the exponential density structure. Normally the contributions to $\bar{J}$ at a given point do not have very localized sources. The discretiza- 


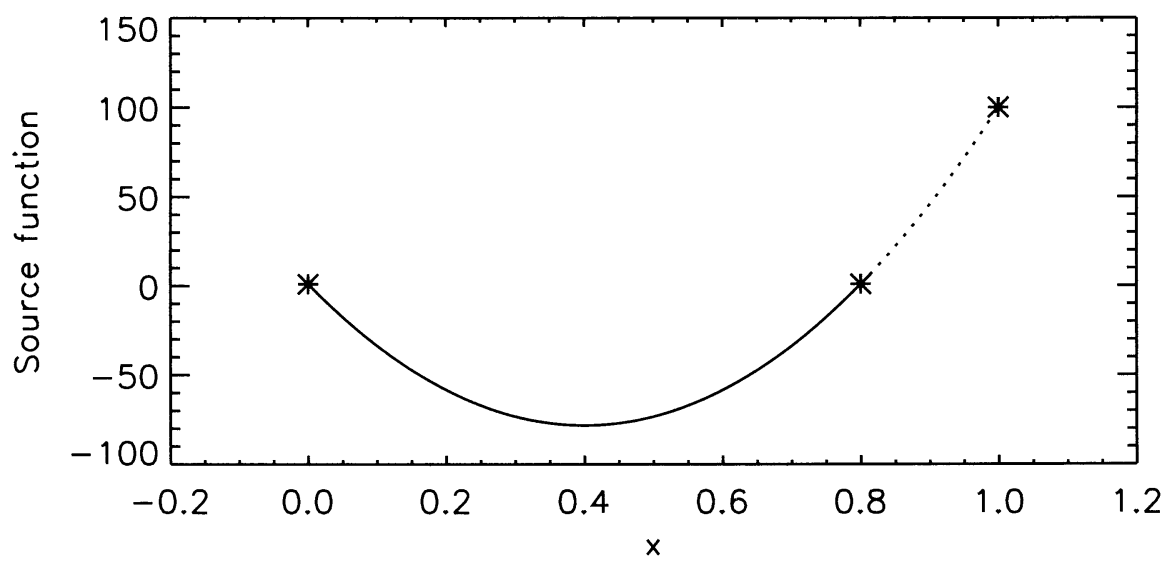

Figure 3. Interpolation "overshoot" in a short characteristic method. The source function in the "Upwind" point $($ at $\mathrm{x}=0.0)$ and 0 -point $(\mathrm{x}=0.8)$ are equal $(=1.0)$ with the "Downwind" point $(\mathrm{x}=1.0)$ much higher $(=100)$. The second order polynomial fit through the three points give negative values in much of the interval between $\mathrm{x}=0$ and $\mathrm{x}=0.8$ even though the source function is positive everywhere leading to an unphysical negative contribution to the intensity in this interval.

tion along given directions will still give artifacts but these can be minimized by changing the ray directions from time-step to time-step.

We have discussed finite difference methods, dividing the computational domain into grid-points. In addition there are finite element methods (e.g. Richling et al. 2001) and Monte Carlo methods (e.g. Auer 2002b, Kurosawa et al. 2002) in current use. Especially the Monte Carlo methods look promising in that they lend naturally to parallelization and photons will be naturally created where there are strong sources, even if they are small in size, relieving problems of resolution in angle.

Methods to solve the 3D NLTE diagnostic problem (i.e. with given atmospheric structure) were thus developed in the late 70's. Computers were then not powerful enough to address realistic problems in 3D. Such problems were, however, computationally feasible some ten years later but, in spite of this fact, until now we have not seen many applications in the literature. The reason is that a $3 \mathrm{D}$ diagnostic NLTE calculation needs as input a realistic $3 \mathrm{D}$ model atmosphere and those are only recently becoming more generally available. A realistic 3D model atmosphere is inherently time dependent and very computationally demanding, even assuming LTE. We address these questions in the next section. 


\section{3D NLTE Structure}

The 3D model atmosphere problem is computationally demanding when a realistic treatment of non-local effects of radiation is attempted. The formal solution of the radiative transfer equation dominates the computational effort and a large number of timesteps have to be performed to cover typical hydrodynamical timescales. The radiative energy balance is determined through an integral over frequency of the radiative cooling term and in 1D atmospheric modelling a very large number of frequencies is normally included to accurately describe the effects of spectral lines on the energy balance (see other contributions in these proceedings). In 3D this approach is not possible and statistical methods need to be employed to decrease the number of frequencies where the transfer equation has to be solved.

Nordlund (1982) decreased the number of frequencies used in LTE radiation hydrodynamic modelling of the Solar convection by combining Opacity Distribution Functions with group mean opacities (see also Stein \& Nordlund 2002). Only four opacity bins describe the effects of radiative heating/cooling in the Solar photosphere remarkably well. Skartlien (2000) generalized the group mean opacity method of Nordlund to also take into account coherent scattering in continua and lines. Skartlien's group mean opacity approach with four bins give heating/cooling rates within $10 \%$ of those calculated from the detailed transfer at 368 frequencies.

Skartlien, Stein, \& Nordlund (2000) used the generalized group mean opacity approach of Skartlien to perform the first self-consistent NLTE Radiation Hydrodynamic simulations of the Solar chromosphere. The treatment needs to be improved since the simulations assume coherent scattering, there is no simultaneous solution of the rate equations, non-equilibrium ionization is ignored and many of the important cooling processes of the chromosphere found in 1D simulations (Carlsson \& Stein 1993, 1994, 1995, 1997, 2002) are lacking. The response of the chromosphere to waves excited by the convection may therefore be rather schematic. The simulations do show, however, how chromospheric shock waves can be excited by collapsing granules and they also show that $3 \mathrm{D}$ NLTE Radiation Hydrodynamic simulations of stellar atmospheres are becoming feasible.

Generalizing 3D LTE radiation hydrodynamic simulations by adding coherent scattering only increases the computational efforts by a factor less than 10. Increasing computational power along the lines of Moore's law (doubling of the CPU power every 18 months) obviously makes possible even more realism. Among effects that one would like to include are effects of Doppler shifts of narrow spectral lines missed by the ODF and multi-group opacity approaches, effects of time dependent ionization of hydrogen (see Carlsson \& Stein 2002), time dependent molecular dissociation equilibria and proper NLTE rate equations for important species. With proper attention to memory issues this is all possible now or in the near future. 


\section{3D NLTE Diagnostics}

In the diagnostic problem, the atmosphere is assumed known. In practice this may be a snapshot from a 3D LTE convection simulation. As shown above even 3D NLTE model atmosphere simulations are now feasible but only with a very simplified treatment of the radiation (hopefully describing sufficiently well, in a statistical sense, the effects of the radiation on the energy balance). For comparisons with observations it is not enough with this statistical description of the radiation and we need to solve the diagnostic problem with a more accurate treatment of the radiative transfer.

Compared with the solution of the $1 \mathrm{D}$ problem, the 3D problem will increase the computational needs by at least a factor $N_{x} \times N_{y}$ where $N_{x}$ and $N_{y}$ are the number of grid cells in the horizontal directions. In practice the increase in computing time is even larger because of the needs for interpolations, more angles and very dense grids in parts of the computational domain in order to resolve structures in other parts. With minimum values of the horizontal dimensions the CPU requirement is at least a factor of 1000 larger than for the $1 \mathrm{D}$ problem. Assuming the formal solution dominates the computational effort we can thus afford a factor of 1000 less formal solutions meaning about a factor of 1000 fewer radiative transitions in our model atom. Alternatively, with a doubling of the CPU power every 18 months, one will have to wait 15 years to achieve in 3D what was earlier the limit in 1D. With limits also on memory (where the development is not as rapid as in CPU speed) this may be an over-optimistic view. To give an idea of what is practically possible today we will give some numbers relating to one of the 3D NLTE codes available, MULTI3D (Botnen \& Carlsson 1999). Other 3D NLTE codes are A-MAZE (Folini \& Walder 1999, Walder \& Folini 2000), the MALI/MUGA code developed by Fabiani Bendicho \& Trujillo Bueno (1999) and RHSC3D from Han Uitenbroek (http://www.sunspot.noao.edu/sunspot/ppages/uitenbr/rh.html)

MULTI3D is a generalization of the 1D code MULTI (Carlsson 1986). The code operates fully in the observer's frame. The statistical equilibrium equations are linearized following Scharmer \& Carlsson (1985). The formal solution is performed using the method of short characteristics allowing for irregular grids in all three directions. The approximate lambda operator is the diagonal operator of Rybicki \& Hummer (1991) and periodic boundary conditions are employed in the horizontal directions.

The memory usage is a critical issue often limiting the problem size. The memory requirement is dominated by the storage of the source function, mean intensity (both proportional to $N_{\nu} \times N_{x} \times N_{y} \times N_{z}$ ) and the approximate lambda matrix (proportional to $N_{L} \times N_{L} \times N_{x} \times N_{y} \times N_{z}$ ), where $N_{\nu}$ is the number of frequency points and $N_{L}$ is the number of atomic energy levels. With no special memory handling $N_{\nu}$ corresponds to the total number of frequency points for all the transitions treated in detail. In MULTI3D the transitions are treated one at a time and $N_{\nu}$ refers to the maximum number of frequency points in one transition, with data for the separate transitions kept on disk when not needed. This represents a major saving in memory usage. Figure 4 (left panel) shows the memory requirement in MULTI3D as a function of $N_{x}=N_{y}$ for a six-level calcium problem. Even such a small model atom requires about $1 \mathrm{~GB}$ of storage for a reasonable size computational box. With $N_{\nu}$ typically on the order of 50 

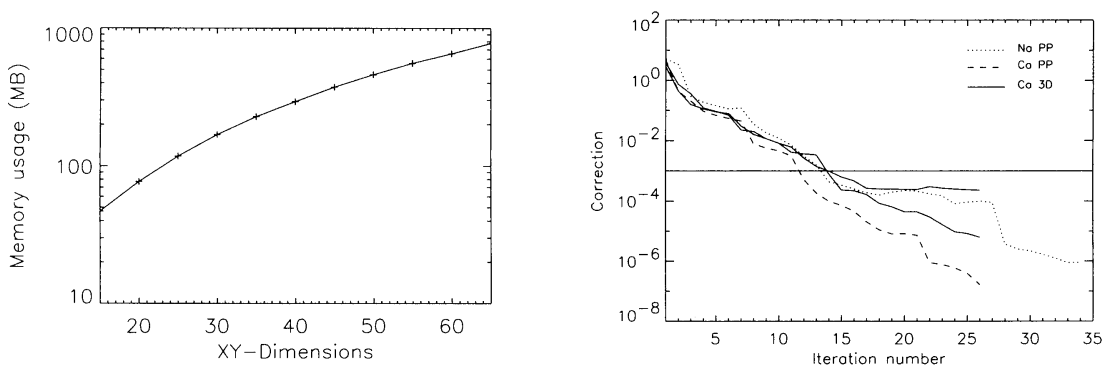

Figure 4. Memory requirement as function of number of grid-points in each horizontal direction (left panel) for a 6-level calcium problem. The number of vertical points is 100 and the number of frequencies in each of the $6 \mathrm{bb}$ transitions is 51 . The right panel shows the convergence behaviour. The two plane-parallel (PP) models, 12 level Na and 6 level $\mathrm{Ca}$, both converge to $10^{-6}$ and beyond. One of the full 3D atmospheres do not converge below $2.310^{-4}$ due to interpolation inaccuracies. From Botnen \& Carlsson (1999)

the memory usage is dominated by the approximate lambda matrix as soon as $N_{L}$ is larger than 10 with this scheme of storage. The approximate lambda matrix storage can easily be domain decomposed in a parallel implementation making possible the solution of large problems.

The convergence behaviour of a number of test problems is given in Figure 4 (right panel). The 1D test problems using the 3D code give the same answers as with the $1 \mathrm{D}$ code and the convergence is unproblematic. When using $3 \mathrm{D}$ snapshots from convection simulations, the convergence is often substantially slower. The reason is that with a $1 \mathrm{D}$ atmosphere it is possible to optimize the grid to avoid large jumps in optical depth and source function. In $3 \mathrm{D}$ this is not so easy since large jumps have to be avoided for all rays. In practice this is impossible and the resulting interpolation errors prevent good accuracy in the solution. Higher order methods with a control of interpolation overshoot would help (Auer 2002a). The convergence is also affected since we need many iterations to propagate the knowledge of the errors across the grid. Multi-grid methods would here help (Fabiani Bendicho et al. 1997).

With MULTI3D it is possible to solve the diagnostic problem for atomic models up to about 20 energy levels on large workstations. An example is the determination of lithium abundances in metal poor stars using $3 \mathrm{D}$ convection simulations as the atmospheric input (Asplund, Carlsson, \& Botnen 2003). A 21-level atomic model with 70 bound-bound transitions and 20 bound-free transitions was used (identical to the one employed in the 1D NLTE studies of Carlsson et al. 1994). The atmospheric model came from 3D LTE convection simulations with $100^{2} \times 82$ grid-points that were resampled to $25^{2} \times 100$ grid-points for the $3 \mathrm{D}$ NLTE diagnostic solutions. Figure 5 shows the comparison of the LTE and NLTE results. In LTE the resonance line of lithium is strongest above granules because of the temperature contrast reversal in the convectively stable layers resulting in low temperature above the hot granules and consequently a large 
population density of neutral lithium. In NLTE quite the opposite happens: the line is weaker above the hot granules because of higher ionization through the strong radiation field. The population densities are thus primarily controlled by the radiation field rather than by the local temperature. The overall equivalent width is substantially smaller in NLTE than in LTE. This shows that for a proper abundance analysis of lithium it is important to employ a full 3D NLTE analysis of a 3D model atmosphere. All other combinations (1D LTE, 1D NLTE, 3D LTE) miss out important processes. This will also be the case for other elements where the non-local radiation field is important, e.g. for other elements with spectral lines from the non-dominant ionization stage.

The limiting factor in problem size on a workstation is largely the amount of memory available. Going to substantially larger atomic models and model atmosphere grids would require better memory optimization and use of massively parallel computers. The latter requires domain decomposition of the computational domain to keep the data local.

\section{Conclusions}

3D NLTE radiative transfer methods of today allow the solution of NLTE problems with moderately large model atoms (20-50 energy levels) for a given 3D model atmosphere (the "diagnostic" problem). To ease the constraints on model atom size and model atmosphere grid size more work is needed on memory optimization, massive parallelism and data locality. 3D NLTE effects on abundance determinations are likely to be important. Such studies are feasible today and should have high priority.

Determining the 3D atmospheric structure including NLTE radiation is several orders of magnitude more computationally demanding than solving the corresponding diagnostic problem. Such 3D NLTE radiation hydrodynamics simulations are only possible with a very limited number of frequency points. Multi-group opacity methods make it possible to carry out such studies with reasonable realism in the effects of radiative transfer on the energy balance. More work should be put into developing approximate methods to describe the NLTE radiative losses in outer stellar atmospheres to enable more realistic structure calculations.

Acknowledgments. This work was supported by grant $146467 / 420$ from the Norwegian Research Council, "Solar and Heliospheric Plasmas".

\section{Discussion}

NISSEN: Do the 3D - non-LTE computations for two in metal-poor main sequence stars with $5700<T_{\text {eff }}<6500 \mathrm{~K}$ preserve the $1 \mathrm{D}$ "Spite plateau" as a function of $T_{\text {eff }}$ ?

CARLSSON: The calculations have so far only been performed for two stars with both showing the same abundance in 3D-NLTE as in 1D-LTE, but since there are large cancelling effects of 3D and NLTE any conclusion is premature with only two stars analyzed. 


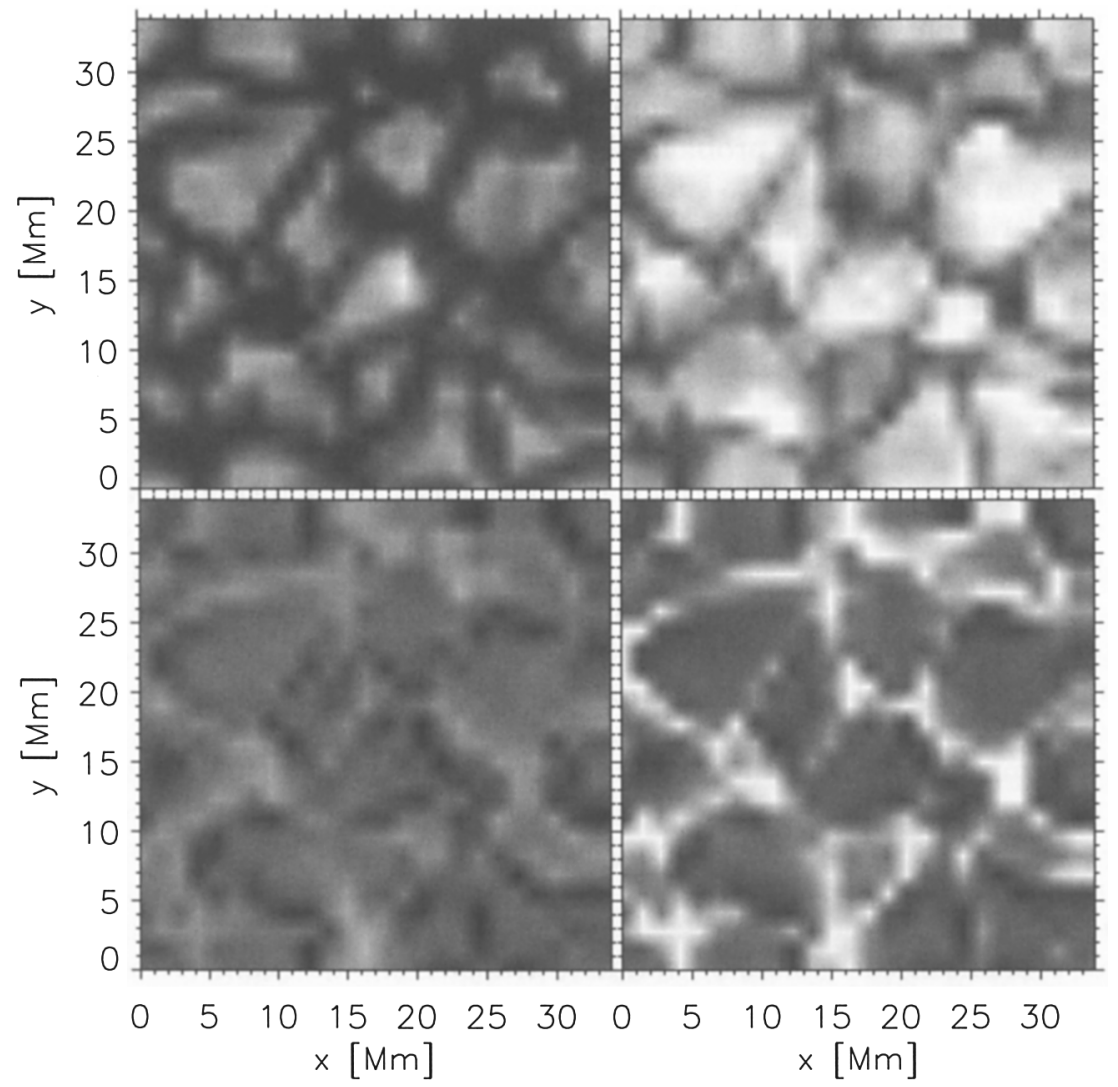

Figure 5. The granulation pattern in one snapshot of HD 140283 seen in disk-center continuum intensity (upper left panel) and equivalent width of the Li I $670.8 \mathrm{~nm}$ line in LTE (upper right panel) and non-LTE (lower left panel); the equivalent width images have the same relative intensity scale to emphasize the overall difference in line strengths. Also shown is the ratio of the non-LTE and LTE equivalent widths (lower right panel). 
STEE: Do you think that it is possible to couple hydrodynamics and radiative transfer equations. Which method of 3D NLTE radiative transfer is better for this coupling? Is it possible to apply this scheme to radiative winds?

CARLSSON: It is possible to couple hydrodynamics and radiative transfer with group mean opacity methods as in the models by Nordlund \& Stein (LTE) and Skartlien (scattering included). Full 3D NLTE transfer is soon possible for very simple model atoms. For winds I am no expert and do not know.

TRAMPEDACH: Are non-LTE effects on the atmosphere structure important in $3 \mathrm{D}$, under the same circumstances as in $1 \mathrm{D}$ ?

CARLSSON: Basically, yes, but because of the increased horizontal transfer possible in $3 \mathrm{D}$ my guess is that NLTE will be more important in 3D than in $1 \mathrm{D}$.

WOITKE: You mention that you have consistent models for the solar chromosphere, including wave-heating and radiative cooling, maybe in non-LTE. What temperature structure do you get from these models? How do these results fit to elder semi-empirical models, where the T-structure was prescribed?

CARLSSON: The 3D NLTE models include scattering, but not detailed NLTE rates nor the effects of slow rates in hydrogen. The $3 \mathrm{D}$ models do, however, support the 1D radiation hydrodynamic simulations with strong hot shocks and most of the time no temperature rise below $1 \mathrm{MeV}$. Semi-empirical models are fundamentally different and the solar chromosphere has to be described with dynamics included. Variations are so large that the mean structure is meaningless.

\section{References}

Asplund, M., Carlsson, M., Botnen, A. V. 2003, ApJ, (submitted)

Auer, L. 2002a, in Workshop on Stellar Atmosphere Modeling, Abstracts from a conference held 8-12 April 2002 in Tuebingen, Germany. Editors: I. Hubeny, D. Mihalas, K. Werner. To be published in the ASP Conference Series, 2003.

Auer, L. 2002b, in Workshop on Stellar Atmosphere Modeling, Abstracts from a conference held 8-12 April 2002 in Tuebingen, Germany. Editors: I. Hubeny, D. Mihalas, K. Werner. To be published in the ASP Conference Series, 2003.

Avery, L. W. 1969, Ph.D. Thesis

Avery, L. W., House, L. L. 1969, Solar Phys., 10, 88

Avery, L. W., House, L. L., Skumanich, A. 1969, J. Quant. Spectrosc. Radiat. Transfer, 9,519

Avrett, E. H., Loeser, R. 1971, J. Quant. Spectrosc. Radiat. Transfer, 11, 573

Botnen, A., Carlsson, M. 1999, in S. M. Miyama, K. Tomisaka,, T. Hanawa (eds.), ASSL Vol. 240: Numerical Astrophysics, Kluwer Academic Publishers, 379

Brandt, A. 1977, Math. Comp., 31, 333

Cannon, C. J. 1970, ApJ, 161, 255 
Cannon, C. J. 1971a, Solar Phys., 16, 314

Cannon, C. J. 1971b, Solar Phys., 21, 82

Cannon, C. J. 1973, ApJ, 185, 621

Cannon, C. J. 1976, A\&A, 52, 337

Carlsson, M. 1986, A Computer Program for Solving Multi-Level Non-LTE Radiative Transfer Problems in Moving or Static Atmospheres, Report No. 33, Uppsala Astronomical Observatory

Carlsson, M., Rutten, R. J., Bruls, J. H. M. J., Shchukina, N. G. 1994, A\&A, 288, 860

Carlsson, M., Stein, R. F. 1993, Proc. Mini-Workshop on Wave Phenomena in Solar Terrestrial Plasmas, Institute of Theoretical Astrophysics, Oslo, p. 21

Carlsson, M., Stein, R. F. 1994, in M. Carlsson (ed.), Proc. Mini-Workshop on Chromospheric Dynamics, Institute of Theoretical Astrophysics, Oslo, p. 47

Carlsson, M., Stein, R. F. 1995, ApJ, 440, L29

Carlsson, M., Stein, R. F. 1997, ApJ, 481, 500

Carlsson, M., Stein, R. F. 2002, ApJ, 572, 626

Fabiani Bendicho, P., Trujillo Bueno, J. 1999, in K. N. Nagendra, J. O. Stenflo (eds.), Solar Polarization, ASSL Vol. 243, Kluwer Academic Publishers, p. 219

Fabiani Bendicho, P., Trujillo Bueno, J., Auer, L. 1997, A\&A, 324, 161

Feautrier, P. 1964, C.R. Acad. Sci. Paris, 258, 3189

Folini, D., Walder, E. 1999, in IAU Symp. 193: Wolf-Rayet Phenomena in Massive Stars and Starburst Galaxies, Vol. 193, p. 352

Hackbush, W. 1985, Multi-Grid Methods and Applications, Springer Verlag, Berlin

House, L. L., Avery, L. W. 1969, J. Quant. Spectrosc. Radiat. Transfer, 9, 1579

Hubeny, I. 1992, in U. Heber, C. S. Jeffery (eds.), The Atmospheres of EarlyType Stars, Lecture Notes in Physics 401, Springer, p. 377

Hubeny, I., Mihalas, D., Werner, K. (eds.) 2003, Stellar Atmosphere Modeling, ASP Conf. Series

Jones, H. P. 1970, Ph.D. Thesis

Jones, H. P. 1971, ApJ, 164, 341

Jones, H. P. 1973a, BAAS, 5, 274

Jones, H. P. 1973b, ApJ, 185, 183

Jones, H. P., Skumanich, A. 1973, ApJ, 185, 167

Kiselman, D., Nordlund, A. 1995, A\&A, 302, 578

Kunasz, P., Auer, L. H. 1988, J. Quant. Spectrosc. Radiat. Transfer, 39, 67

Kurosawa, R., Hillier, D. J., Pittard, J. M. 2002, A\&A, 388, 957

Mihalas, D. 1978, Stellar Atmospheres, W. H. Freeman and Co., San Francisco (second edition)

Mihalas, D., Auer, L. H., Mihalas, B. R. 1978, ApJ, 220, 1001 
Nakamoto, T. 1999, in S. M. Miyama, K. Tomisaka, T. Hanawa (eds.), ASSL Vol. 240: Numerical Astrophysics, Kluwer Academic Publishers, p. 375

Nordlund, A. 1982, A\&A, 107, 1

Nordlund, A. 1985, in J. E. Beckman, L. Crivellari (eds.), Progress in stellar spectral line formation theory, NATO ASI Series C152, Reidel, Dordrecht, p. 215

Olson, G. L., Auer, L. H., Buchler, J. R. 1986, J. Quant. Spectrosc. Radiat. Transfer, 35, 431

Olson, G. L., Kunasz, P. 1987, J. Quant. Spectrosc. Radiat. Transfer, 38, 325

Richling, S., Meinköhn, E., Kryzhevoi, N., Kanschat, G. 2001, A\&A, 380, 776

Rybicki, G. B., Hummer, D. G. 1991, A\&A, 245, 171

Scharmer, G. B. 1981, ApJ, 249, 720

Scharmer, G. B., Carlsson, M. 1985, J. Comput. Phys., 59, 56

Skartlien, R. 1998, Ph.D. Thesis

Skartlien, R. 2000, ApJ, 536, 465

Skartlien, R., Stein, R. F., Nordlund, Å. 2000, ApJ, 541, 468

Stein, R. F., Nordlund, A. 2002, in Workshop on Stellar Atmosphere Modeling, Abstracts from a conference held 8-12 April 2002 in Tuebingen, Germany. Editors: I. Hubeny, D. Mihalas, K. Werner. To be published in the ASP Conference Series, 2003.

Steiner, O. 1991, A\&A, 242, 290

Stenholm, L. G. 1977, A\&A, 54

Stenholm, L. G., Stenflo, J. O. 1977, A\&A, 58, 273

Stenholm, L. G., Stenflo, J. O. 1978, A\&A, 67, 33

Trujillo Bueno, J., Fabiani Bendicho, P. 1995, ApJ, 455, 646

van Noort, M., Hubeny, I., Lanz, T. 2002, ApJ, 568, 1066

Väth, H. M. 1994, A\&A, 284, 319

Walder, R., Folini, D. 2000, in ASP Conf. Ser. 204: Thermal and Ionization Aspects of Flows from Hot Stars, p. 281 